\title{
Development and bin mapping of a Rosaceae Conserved Ortholog Set (COS) of markers
}

\author{
Antonio Cabrera1 ${ }^{1}$, Alex Kozik², Werner Howad ${ }^{3}$, Pere Arus ${ }^{3}$, Amy F Iezzoni ${ }^{4}$ \\ and Esther van der Knaap*1
}

\begin{abstract}
Address: ${ }^{1}$ Department of Horticulture and Crop Science, The Ohio State University/Ohio Agricultural Research and Development Center, Wooster OH 44691, USA, ${ }^{2}$ Genome Center and Department of Plant Sciences, University of California, Davis, California 95616, USA, ${ }^{3}$ Departament de Genètica Vegetal, Laboratori de Genètica Molecular Vegetal, CSIC-IRTA, 08348 Cabrils, Spain and ${ }^{4}$ Department of Horticulture, Michigan State University, East Lansing MI 48824, USA
\end{abstract}

Email: Antonio Cabrera - cabrera.16@buckeyemail.osu.edu; Alex Kozik - akozik@atgc.org; Werner Howad - werner.howad@irta.es; Pere Arus - pere.arus@irta.es; Amy F Iezzoni - iezzoni@msu.edu; Esther van der Knaap* - vanderknaap.1@osu.edu

* Corresponding author

Published: 29 November 2009

BMC Genomics 2009, 10:562 doi:10.1186/147|-2/64-10-562
Received: 19 August 2009

Accepted: 29 November 2009

This article is available from: http://www.biomedcentral.com/I47/-2/64/I0/562

(C) 2009 Cabrera et al; licensee BioMed Central Ltd.

This is an Open Access article distributed under the terms of the Creative Commons Attribution License (http://creativecommons.org/licenses/by/2.0), which permits unrestricted use, distribution, and reproduction in any medium, provided the original work is properly cited.

\begin{abstract}
Background: Detailed comparative genome analyses within the economically important Rosaceae family have not been conducted. This is largely due to the lack of conserved gene-based molecular markers that are transferable among the important crop genera within the family [e.g. Malus (apple), Fragaria (strawberry), and Prunus (peach, cherry, apricot and almond)]. The lack of molecular markers and comparative whole genome sequence analysis for this family severely hampers crop improvement efforts as well as QTL confirmation and validation studies.
\end{abstract}

Results: We identified a set of 3,818 rosaceaous unigenes comprised of two or more ESTs that correspond to single copy Arabidopsis genes. From this Rosaceae Conserved Orthologous Set (RosCOS), 1039 were selected from which 857 were used for the development of intron-flanking primers and allele amplification. This led to successful amplification and subsequent mapping of 613 RosCOS onto the Prunus TxE reference map resulting in a genome-wide coverage of 0.67 to 1.06 gene-based markers per CM per linkage group. Furthermore, the RosCOS primers showed amplification success rates from 23 to $100 \%$ across the family indicating that a substantial part of the RosCOS primers can be directly employed in other less studied rosaceaous crops. Comparisons of the genetic map positions of the RosCOS with the physical locations of the orthologs in the Populus trichocarpa genome identified regions of colinearity between the genomes of Prunus-Rosaceae and Populus-Salicaceae.

Conclusion: Conserved orthologous genes are extremely useful for the analysis of genome evolution among closely and distantly related species. The results presented in this study demonstrate the considerable potential of the mapped Prunus RosCOS for genome-wide marker employment and comparative whole genome studies within the Rosaceae family. Moreover, these markers will also function as useful anchor points for the genome sequencing efforts currently ongoing in this family as well as for comparative QTL analyses. 


\section{Background}

The Rosaceae is an important plant family that includes more than 90 genera and 3000 species. The family belongs to the Rosid clade and is closely related to the Salicaceae (including poplar), Leguminoseae (including Medicago and soybean), Cucurbitaceae (including cucumber and melon) and more distantly related to the Brassicaceae (including Arabidopsis). The Rosaceae is divided into three subfamilies, two of which include some of the most economically important temperate fruit crops [1]. The largest subfamily is the Spiraoideae to which Malus (apple), Pyrus (pear) and Prunus (peach, cherry, almond, apricot) belong. The second largest subfamily is the Rosoideae to which Fragaria (strawberry), Rubus (currants, blackberries, raspberries) and Rosa (rose) belong. Within the family, apple, peach and strawberry have been utilized as model species for Rosaceae biology, genetics and genomics [2].

Comparative analyses of plant genomes offer insights into genome evolution and speciation of closely as well as more distantly related species. In particular, knowledge of the extent and locations of syntenic blocks and chromosomal rearrangements enables the transfer of genomic information among species. This information would aid genome-wide as well as targeted marker development for the identification and validation of loci controlling traits that are important for crop improvement. Without the availability of several sequenced plant genomes within one family, comparative analyses often rely on molecular markers that are shared among the species. One of the earliest efforts towards the construction of comparative plant maps using molecular markers was conducted in the Solanaceae family. Assessment of the degree of similarity between tomato and pepper $[3,4]$ and tomato and potato [5] show that the more closely related species, tomato and potato, underwent fewer rearrangements compared to the more distantly related tomato and pepper. Similarly in the Poaceae family, conservation of large chromosomal regions between wheat, barley and rye genomes have been identified $[6,7]$. The application of comparative sequence analysis within the grasses greatly facilitated the positional cloning of important genes such as VRN1 from wheat, a species for which map-based cloning was deemed impossible due to its large genome size and the presence of many repetitive elements that would hamper chromosome walking efforts [8].

Despite the lack of extensive investigations, the potential for comparative genome analysis within the Rosaceae family has been demonstrated by several studies. Genome colinearity was found among Prunus species [9-16]. These comparative studies were based on the Prunus reference map $(x=8)$, the most detailed genetic map in the
Rosaceae, that is derived from an interspecific almond $(P$. dulcis) cv. Texas $\times$ peach $(P$. persica) cv. Earlygold (abbreviation $\mathrm{TxE}$ ) $\mathrm{F}_{2}$ mapping population [10]. Good colinearity and marker transferability within the family was also demonstrated by the identification of syntenic regions of the Malus and Prunus genomes [9,17], and between the more distant genera Prunus and Fragaria $[18,19]$. However, a comprehensive and extensive comparative map such as those that were constructed in the Solanaceae and Poaceae families has not been achieved for the Rosaceae. This is mostly due to the lack of conserved markers to apply across the entire family $[12,18]$.

Genes that are highly conserved and are present as low or single copy in genomes are particularly useful as markers for genome evolution studies as well as whole genome comparative analyses [20,17]. A Conserved Ortholog Set (COS) is defined as a collection of genes that are conserved in sequence and copy number throughout plant evolution [20]. In contrast, paralogs represent duplicated regions within the genome as a result of single gene duplications and/or large scale polyploidization events [21]. The development of markers from single copy and conserved genes is critical in comparative mapping studies as these markers enable an unambiguous determination of the degree of synteny [22]. In addition, the single copy conserved genes reduce the possibility of erroneously identifying chromosomal rearrangements that could result from mapping paralogous genes [23].

Complete whole-genome sequence information of model plants together with improved genomic resources from other species, such as EST databases, provide the opportunity for the in silico identification of candidate COS. Using the Arabidopsis whole genome sequence and the EST databases of potato, tomato and pepper, Wu et al identified 2869 Solanaceaous COS [21]. Likewise, a universal set of COS markers was developed for the Asteraceae family after comparing EST from sunflower and lettuce against the whole genome of Arabidopsis [24]. Moreover, comparative genome sequence analysis between the three sequenced model species, Arabidopsis thaliana, Oryza sativa and Populus trichocarpa resulted in the identification of 753 COS candidates among the angiosperms of which 55 to 359 could be identified from pairwise comparisons among four gymnosperm EST databases [25]. Once developed, COS markers have been widely employed to link the genomes of related species within families [20,26-29]

In this study, we report the first step towards a comprehensive and dense comparative genetic map for rosaceous species. We present the development of a set of conserved Rosaceae gene-based sequences corresponding to single copy Arabidopsis genes. These Rosaceae COS (RosCOS) 
were subsequently mapped using the bin map population corresponding to the Prunus TxE reference map $[10,30]$. Our analyses show that nearly all of the mapped RosCOS are present once in the Prunus genome suggesting that this genus did not undergo a hitherto unknown recent polyploidization event. Additionally, we compared the genetic location of these RosCOS to the physical location of the poplar and Arabidopsis orthologs. These analyses identified many regions that exhibited synteny between Prunus and poplar and to a lesser extent to Arabidopsis.

\section{Results and Discussion Construction of the RosCOS set}

The Rosaceae ESTs that were publicly available as of December 2007 were used to construct the set of COS. The highest numbers of available Rosaceae ESTs were from Malus, Prunus and Fragaria, totaling up to $97.6 \%$ of all Rosaceae ESTs (Table 1). After comparing these ESTs to Arabidopsis single copy genes, we identified 30,801 putative orthologs (Figure 1). The CAP3 assembly of these ESTs resulted in 7,247 unigenes corresponding to 2,324 single copy Arabidopsis genes. Of these, 3,818 were contigs comprised of at least two ESTs and 3,429 were singletons. On average, the number of unigenes corresponded to 3.1 Rosaceae putative COS per Arabidopsis single copy gene. When we compared the distribution among contigs versus the singletons and the mixture of contigs and singletons, the majority of Arabidopsis single copy genes was represented by up to three Rosaceae unigenes (Figure 2). Also, the data showed that a significant number of the Arabidopsis single copy genes were represented by singletons indicating the lack of sufficiently deep EST data in the Rosaceae to permit assembly into contigs. The apparent redundancy in this unigene dataset is likely due to: 1) ESTs corresponding to the same gene but aligning to different parts of the gene, 2) sufficient nucleotide divergence within the Rosaceae EST from different species such that CAP3 would not allow them to be assembled into the same unigene, 3 ) errors in cloning, sequencing, as well as alternative splicing. Further investigation into the unigene duplicates is provided below.
Due to single pass sequencing of EST clones, the chance of sequencing errors can be considerable. In an effort to avoid the design of primers in regions of poor sequence quality, we focused on the 3,818 unigenes that were represented by at least two ESTs. Moreover, contigs tended to have more sequence information (i.e. longer sequences) which was helpful in the design of primers flanking the predicted intron sites. Each contig was named RosCOS\#\#\# to indicate that this was the set of putatively conserved orthologous Rosaceae sequences. We narrowed the collection down further by selecting RosCOS that were represented by at least two of the three key genera in the family or Prunus alone (see Additional file 1). This selection was chosen to enhance the chance of successful amplification of Prunus DNA with the designed primers because of our goal to map these RosCOS on the Prunus reference map. The reduction led to the final data set of 1,039 RosCOS (Figure 1). We noticed that contigs harboring ESTs from more than one genus usually exhibited a higher number of mismatches in Fragaria than in Malus or Prunus which is consistent with the greater phylogenetic distance between Fragaria and the other two genera [1].

\section{Amplification and mapping of RosCOS in Prunus}

Of the 1,039 RosCOS, 857 were selected for the design of intron-flanking primers because their sequences covered at least one putative intron (Figure 3 ). These primers were used to amplify the corresponding region from the TxE peach parent 'Earlygold', the $\mathrm{F}_{1}$, and the Prunus bin map set that consisted of six $\mathrm{F}_{2}$ individuals. Amplification success and mapping ability was evaluated, which demonstrated that $91 \%$ of the primers amplified Prunus DNA of which only $10 \%$ were monomorphic (Table 2 ). The percentage of RosCOS that exhibited only one SNP was $18 \%$ whereas $43 \%$ harbored at least 2 SNPs. A total of 39\% of the polymorphic RosCOS contained at least one InDel (see Additional file 2 for detailed information about each RosCOS).

A total of 613 RosCOS were assigned to 63 of the 67 Prunus bins (Figure 4). This included six RosCOS for which

Table I: Number of Rosaceae ESTs from different subfamilies and genera.

\begin{tabular}{cccc}
\hline Sub-family' & Genus & Total EST & EST corresponding to Arabidopsis COS \\
\hline Rosoideae & Fragaria & 50,882 & 3,899 \\
Spiraeoideae & Malus & 260,594 & 20,502 \\
Spiraeoideae & Prunus & 91,354 & 5,668 \\
\hline Spiraeoideae & Pyrus & 341 & 8 \\
Rosoideae & Rosa & 9,289 & 712 \\
Rosoideae & Rubus & 323 & 12 \\
Spiraeoideae & Photinia & 44 & 0 \\
\hline & Total & $\mathbf{4 I 2 , 8 2 7}$ & $\mathbf{3 0 , 8 0 1}$ \\
\hline
\end{tabular}

'Classification from Potter et al [I]. 


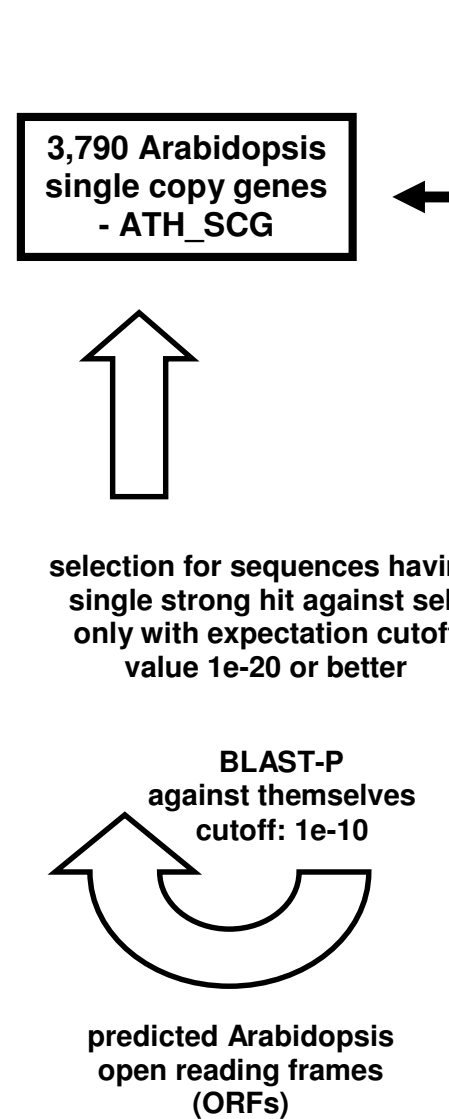

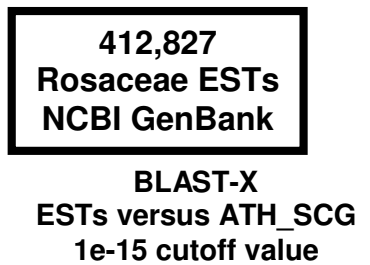

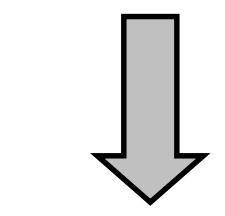

30,801 RosCOS EST candidates

selection of regions corresponding to Arabidopsis ORF

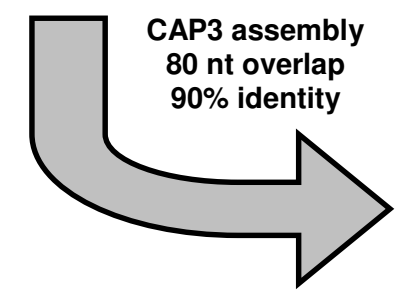

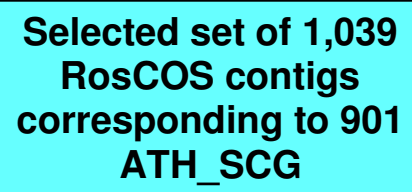

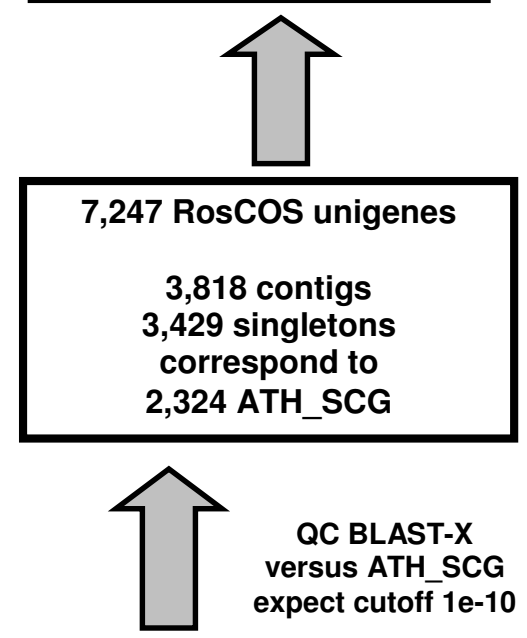

7,573 RosCOS unigene candidates

3,820 contigs 3,753 singletons

Figure I

Identification of Conserved Orthologous Set (COS) of sequences between Arabidopsis and Rosaceae.

the position could not be conclusively identified. For these six, the 'Earlygold' parent and F1 were both heterozygous as were all the F2 progeny individuals (Table 2). The heterozygous genotype found for all six F2 plants comprising the bin population is indicative of the position on the top of linkage group 4. Therefore, we tentatively placed these six RosCOS with the other RosCOS in bin 4:18 (Figure 4). However, it was also possible that these RosCOS represented recent gene duplications as was observed in a few other cases (Howad and Arus, unpubl). In addition, some RosCOS were clearly polymorphic but could not be assigned to an existing bin. The 36 unbinned RosCOS were termed "orphan COS" of which 17 grouped in four distinct bins. The fact that several orphan COS clustered together suggested that these bins correctly represent the Prunus genome; however, the genomic location is unknown. Only $1 \%$ of the RosCOS exhibited ambiguous segregation due to difficulty in scoring the SNPs and associated double peaks in the chromatograms. Six per- cent of the sequencing reactions failed, indicating the overall high quality of the sequence data (Table 2). In all, the average marker density per centimorgan $(\mathrm{cM})$ ranged from 0.67 to 1.06 for the eight Prunus chromosomes (Table 3). Marker density within bins ranged from 0.2 to 18 per $\mathrm{cM}$ which might be indicative of regions of low and high recombination frequencies, respectively.

The marker density per linkage group is high and could be inflated due to the fact that one single copy Arabidopsis gene is represented by, on average, 3.1 Rosaceae unigenes (see above). Among the mapped RosCOS, we noted that 55 Arabidopsis single copy genes corresponded to two or more RosCOS (Table 4, see Additional file 3). Importantly, five of the 55 putatively duplicated Arabidopsis single copy genes mapped to different positions in the Prunus genome, indicating that at least some genes were duplicated in Prunus while they were single copy in Arabidopsis (Table 4 ). The remaining 50 putatively duplicated 


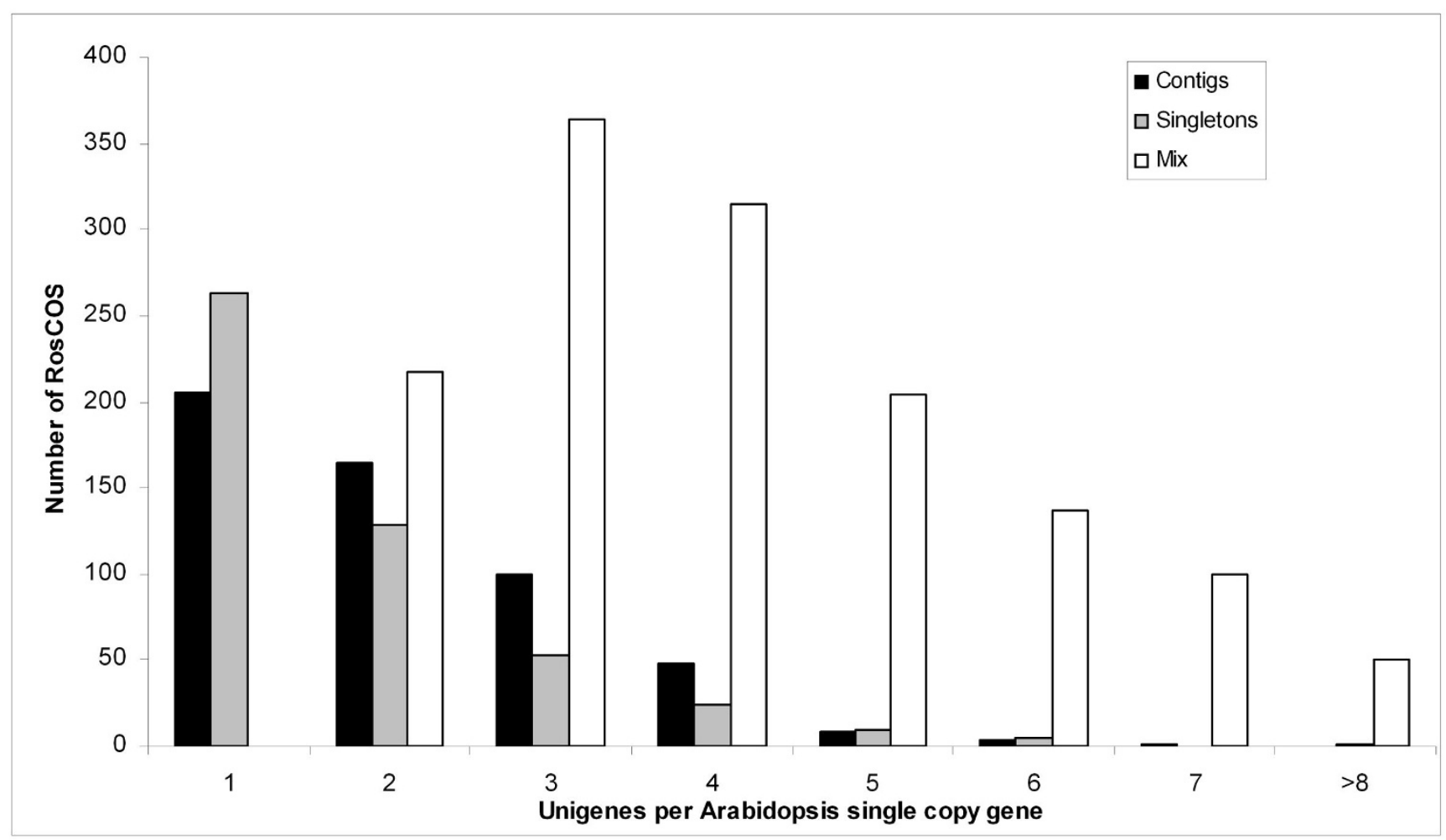

\section{Figure 2}

Rosaceae unigene content per Arabidopsis single copy gene. Numbers on the $\mathrm{X}$-axis represent the number of Rosaceae unigenes matching a unique Arabidopsis single copy gene. Black bars represent unigenes comprised of at least two ESTs (contig); the gray bars represent singletons and white bars represent mixtures of singletons and contigs.

genes mapped to the same bin which implied that these could have been derived from one Rosaceae conserved gene (see Additional file 3). To further address this possibility, a closer examination of the CAP3 assembly of the 50 single copy Arabidopsis genes with more than one RosCOS representative revealed that in 36 cases these RosCOS corresponded to the same region of the Arabidopsis single copy gene. The reason that these unigenes were not assembled into one RosCOS appeared to stem from the fact that the overlapping region was too short and/or too divergent to ensure the assembling into one RosCOS. It is therefore likely that these RosCOS correspond to a single
Rosaceae conserved gene and are not the result of gene duplication. For the remaining 14 putatively duplicated Arabidopsis single copy genes, the corresponding RosCOS did not overlap with the same region of the Arabidopsis gene. Therefore, whether these RosCOS corresponded to the same gene or a tandemly duplicated gene pair could not be determined with the present data. However, despite the evidence of a few duplicated genes, which may have occurred after the divergence of Arabidopsis-Brassicaceae and Rosaceae or represent gene loss in Arabidopsis, these data strongly support the evidence for the lack of a recent large scale genome duplication event in Prunus.

Table 2: Amplification and bin mapping success for 857 RosCOS primer pairs.

\begin{tabular}{cccccc}
\hline & \multicolumn{4}{c}{ Polymorphic RosCOS } & Failed sequence Monomorphic \\
\cline { 2 - 5 } $\begin{array}{c}\text { Amplification in } \\
\text { TxEI }\end{array}$ & Bin mapped & Orphan COS & Putative bin 4:183 & $\begin{array}{c}\text { Inconclusive } \\
\text { segregation }\end{array}$ & M \\
\hline $784(91 \%)$ & $607(78 \%)$ & $36(5 \%)$ & $6(0.8 \%)$ & $8(1 \%)$ & $47(6 \%)$ \\
\hline
\end{tabular}

IPCR amplification success in the TXE mapping population was visualized on agarose gels.

${ }^{2}$ RosCOS markers that mapped in bins that are not reported (orphan bins). Collectively, they represent 23 orphan bins of which 4 are comprised of two or more RosCOS.

${ }^{3}$ Heterozygous RosCOS in all the genotypes of the bin set resemble bin 4:18 on linkage group 4. 


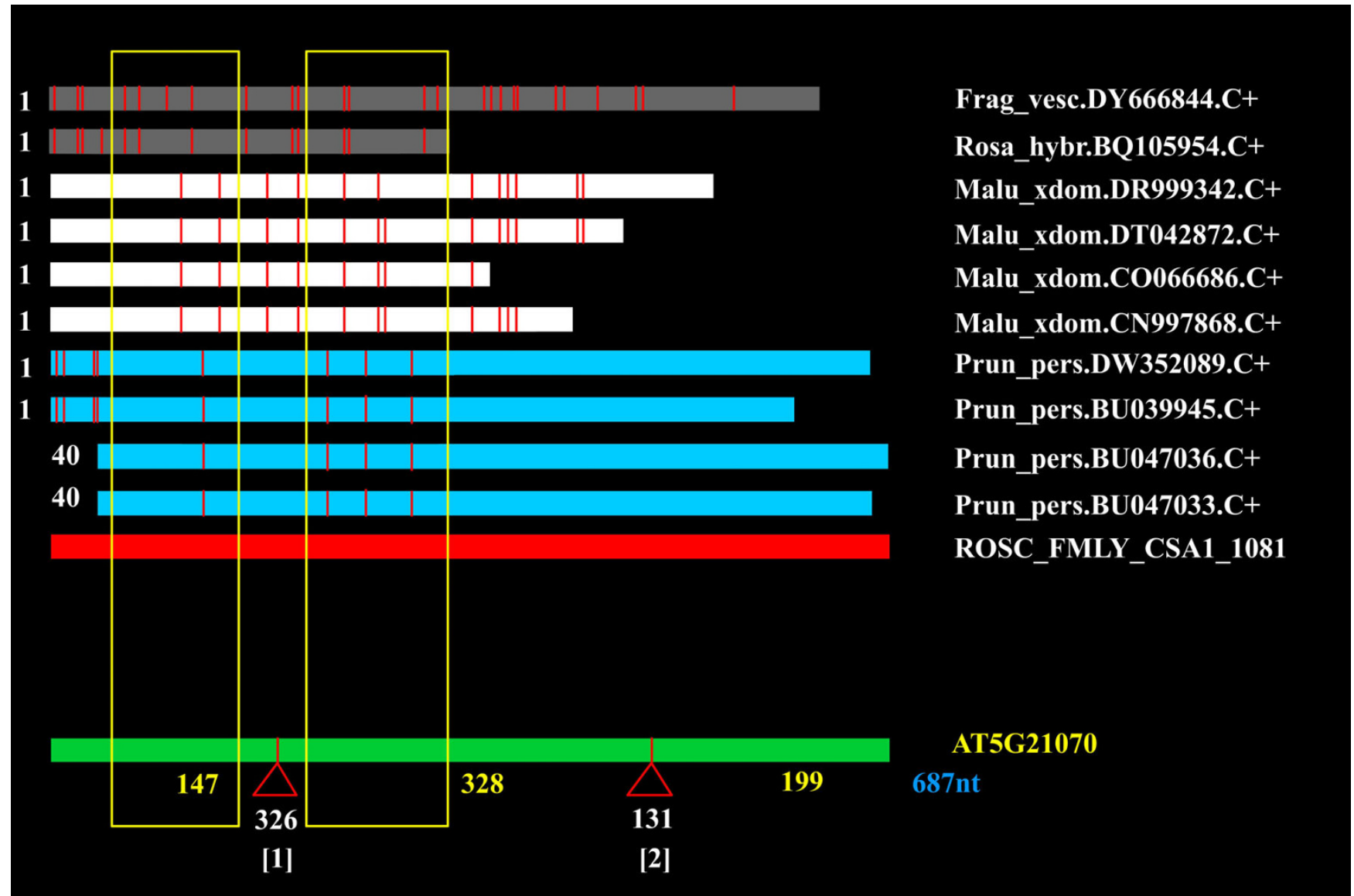

Figure 3

Development of primers in adjacent exons that flank the same intron. The output of the python contig software tool [40] allows the determination of the intron position based on the Arabidopsis genome sequence and EST constitution of RosCOS. The Rosaceae consensus sequence (red), composed of Fragaria (grey), Malus (white) and Prunus (blue) ESTs, and was compared to the Arabidopsis genome (green). The intron $(\Delta)$ and flanking positions (yellow blocks) were used to develop universal primers using Primer3 v. 0.4.0 [4I].

\section{Synteny between Rosaceae, Arabidopsis and Populus}

The availability of the Arabidopsis and poplar genomes allowed us to determine the level of synteny among these species and Prunus. Because gene annotation is more complete for Arabidopsis than any other plant species, the translated Arabidopsis single copy genes corresponding to
RosCOS that mapped to the same TxE bin were searched against the translated poplar genome using the TBLASTN function. After identifying the location in poplar of RosCOS that mapped together in Prunus, we found several syntenic regions between these genomes (Figure 5). Importantly, the mapping of the poplar COS confirmed

Table 3: RosCOS marker density on the eight Prunus TxE linkage groups.

\begin{tabular}{|c|c|c|c|}
\hline Linkage Group & cM length of the linkage group & Number of RosCOS mapped & RosCOS density per cM \\
\hline I & 87.0 & 129 & 0.67 \\
\hline 2 & 50.5 & 69 & 0.73 \\
\hline 3 & 48.4 & 67 & 0.72 \\
\hline 4 & 62.5 & 59 & 1.06 \\
\hline 5 & 49.1 & 67 & 0.73 \\
\hline 6 & 83.7 & 85 & 0.98 \\
\hline 7 & 70.6 & 72 & 0.98 \\
\hline 8 & 55.9 & 65 & 0.86 \\
\hline
\end{tabular}




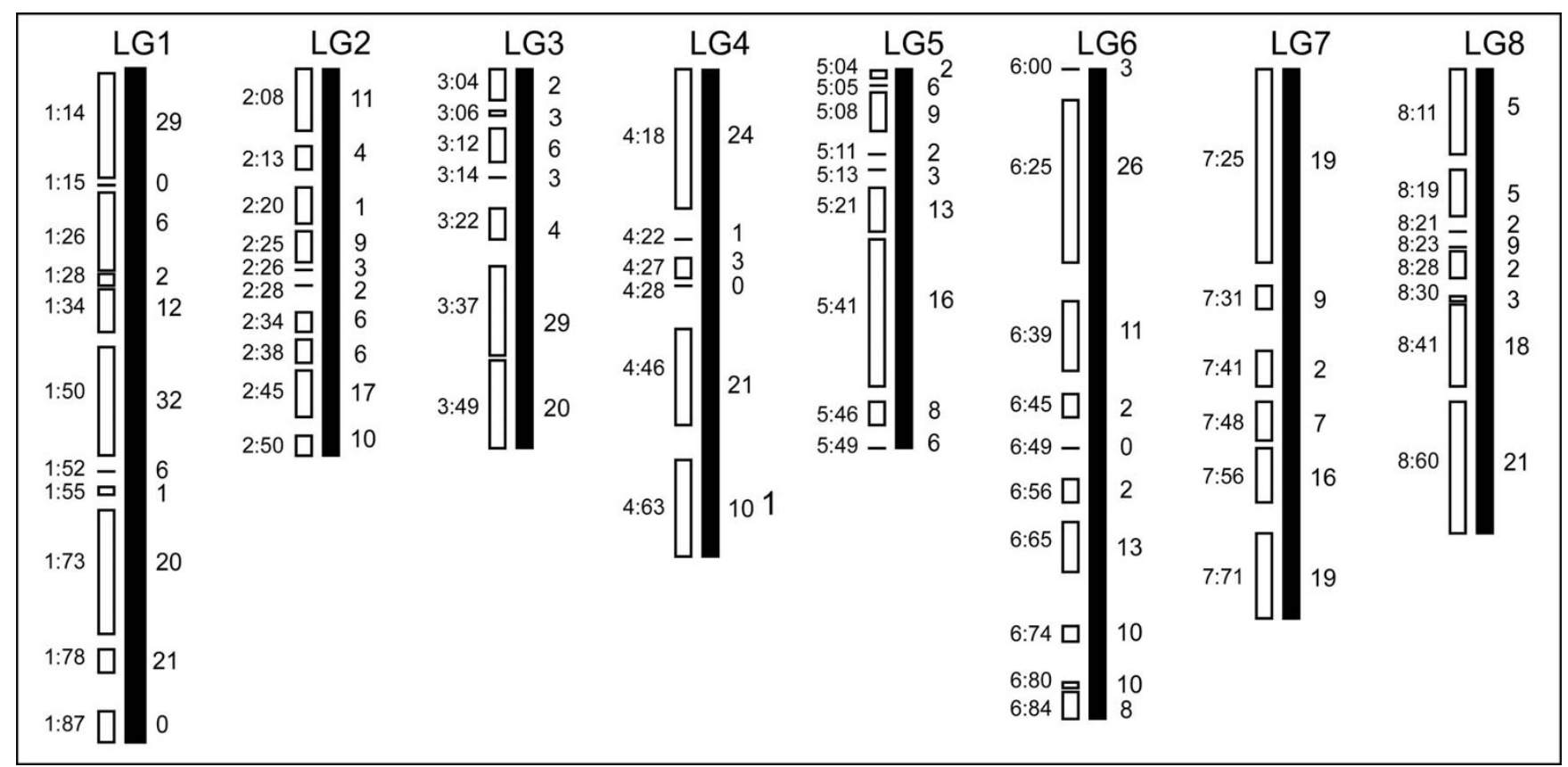

\section{Figure 4}

Position of the 613 RosCOS on the TxE bin map. Thick black vertical lines represent the linkage groups indicated above the lines. The white boxes on the left of each linkage group symbolize the bins (minimum bin length). The number before the semicolon indicates the linkage group and the number following the semicolon indicates the genetic position (in $\mathrm{cM}$ ) of the last marker within the respective bin. Numbers on the right of each linkage group represent the number of RosCOS that map to the bin.

nearly all the previously reported homeologous gene blocks shared by two poplar chromosomes presumed to have arisen from the most recent salicoid wide-genome duplication event [31]. For instance, RosCOS that mapped in the TxE bin 1:34 confirmed duplicated blocks of poplar linkage groups 1 and 3 (Figure 5A). The high level of synteny between poplar and Prunus as well as the conservation of gene order in paralogous regions of the poplar genome strongly supported the potential of the RosCOS for comparative mapping across the Rosaceae family. These results also suggested that the order of the RosCOS in the Rosaceae can be predicted based on their order in poplar, although this would have to be confirmed by genome sequence analysis or higher resolution genetic mapping. The size of the syntenic blocks was defined as large (more than seven RosCOS corresponding to poplar orthologs in a $2 \mathrm{Mb}$ region), medium (harboring five to six RosCOS) and small (harboring three to four RosCOS). As a result, we identified six large syntenic blocks represented by bins $1: 73,8: 41,8: 60$, and the adjoining bins $5: 21$ and $5: 41 ; 6: 65$ and $6: 74$; and $6: 80$ and $6: 84$. In addition, 21 medium and 20 small syntenic blocks were also observed (Figure 5).

We also analyzed the number of RosCOS that mapped to the same Prunus bin and their corresponding position in the Arabidopsis genome. The data indicated that the Arabidopsis -Prunus synteny blocks tended to be smaller compared to the size of the Populus-Prunus blocks (Figure 6). For example, most of the blocks in Arabidopsis had only

Table 4: Number of Arabidopsis single copy genes corresponding to more than one RosCOS and their Prunus bin map co-localization.

\begin{tabular}{cc} 
Number of Arabidopsis genes that correspond more than one & RosCOS bin map locations \\
RosCOS & Map to the same bin Map to separate bins $\quad$ Total \\
\hline 55 & $103(91 \%)$
\end{tabular}

'Five Arabidopsis single copy genes corresponded to two RosCOS that map to different positions in Prunus, indicating possible Prunus gene duplications not found in Arabidopsis. 


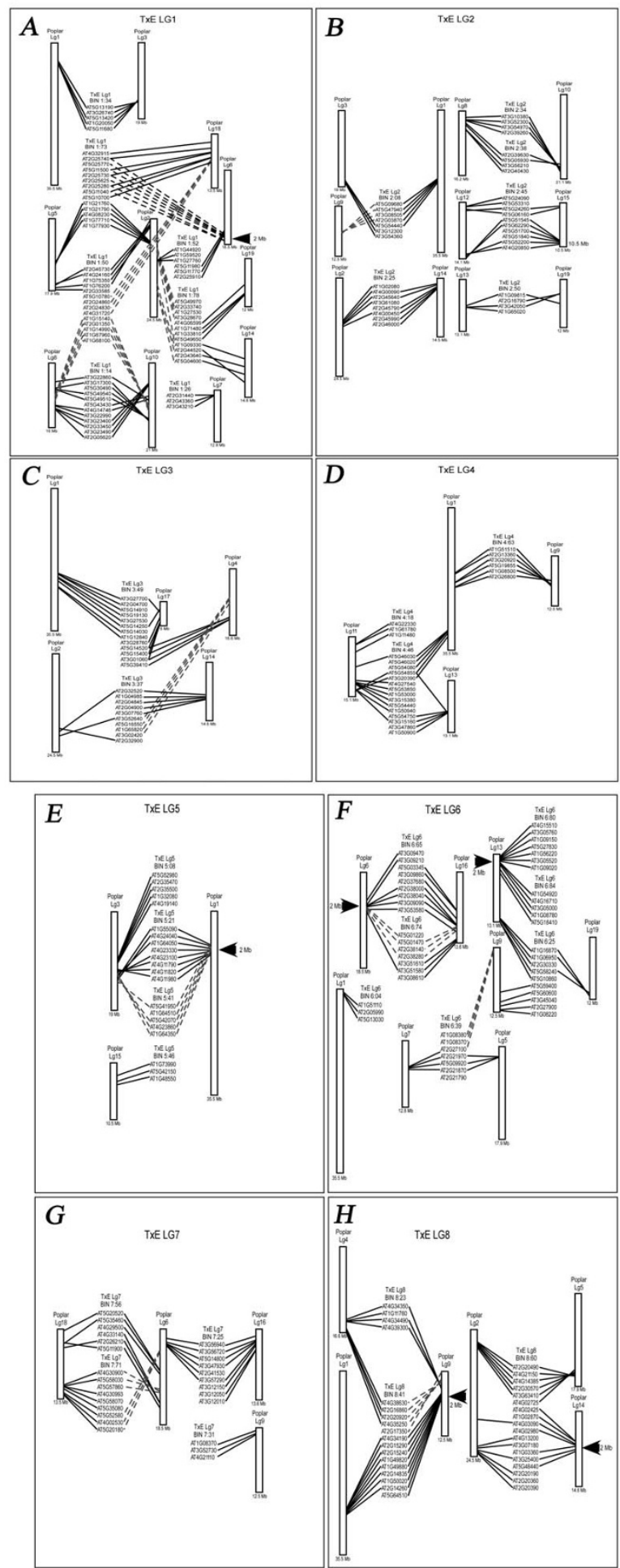

Figure 5

Synteny between Prunus and Populus. Arabidopsis single copy genes corresponding to the bin mapped RosCOS were compared to the poplar genome. RosCOS that mapped to the same bin were selected for synteny analysis when three or more poplar orthologs were within $2 \mathrm{Mb}$ from another for at least one poplar linkage group. Arrows indicate the largest syntenic blocks within $2 \mathrm{Mb}$ of the poplar genome. A through $\mathrm{H}$ represent Prunus linkage groups I through 8 , respectively. 


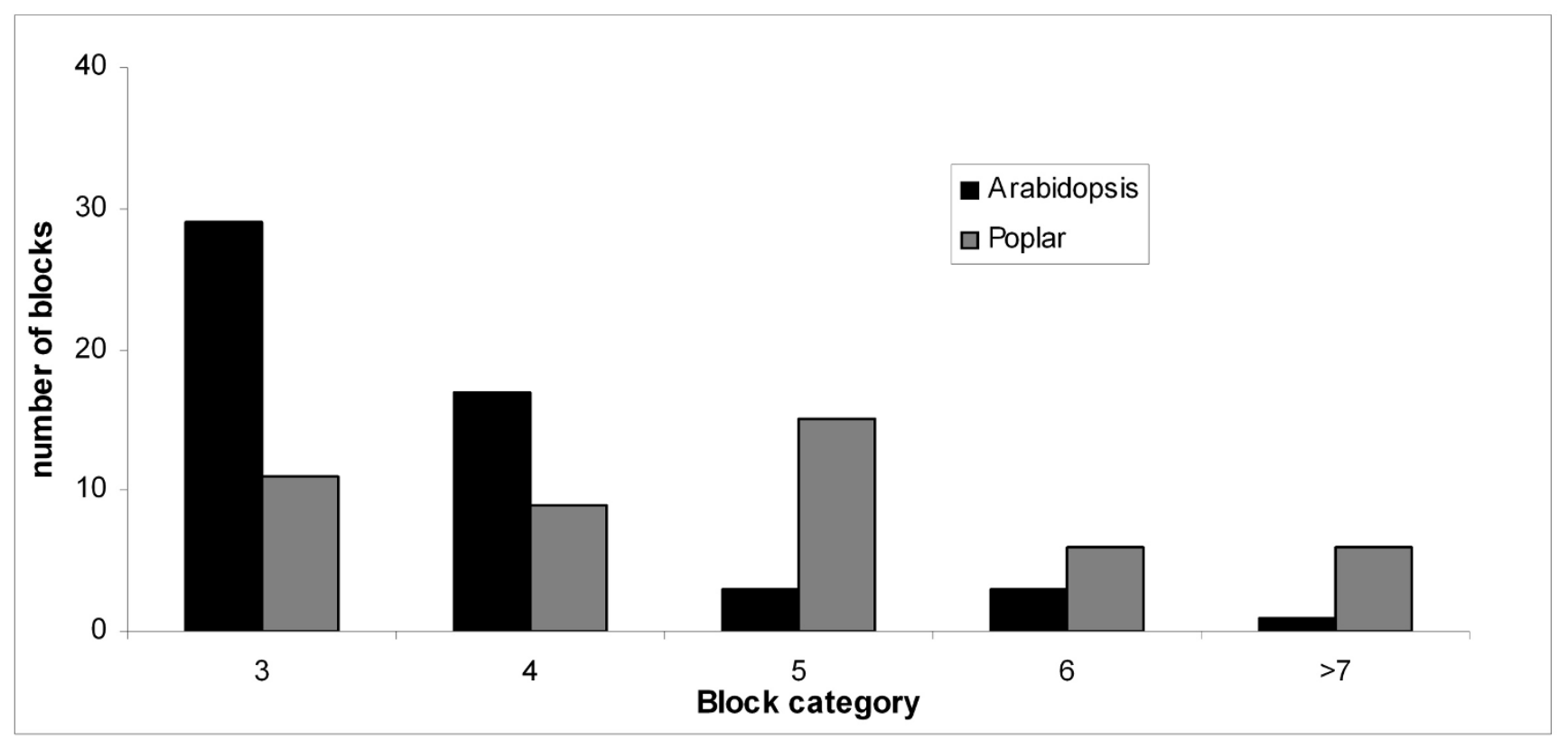

Figure 6

Syntenic block size of Prunus with Arabidopsis and poplar. Numbers on the $X$-axis represent the number of RosCOS that mapped to the same Prunus bin which were also identified within $2 \mathrm{Mb}$ in the Arabidopsis and poplar genomes, black and gray bars, respectively.

three RosCOS within a $2 \mathrm{Mb}$ interval whereas most of the blocks in poplar had five RosCOS within a $2 \mathrm{Mb}$ interval. This result suggested that the order of the Prunus RosCOS is less conserved with that of Arabidopsis compared to poplar. This is an expected finding since Arabidopsis is more distantly related to Rosaceae than is poplar and is consistent with previous findings [32].

\section{Amplification of RosCOS across the Rosaceae}

The transferability of molecular markers across different species is an important feature of conserved orthologous sequences in addition to the common ancestry these sequences represent. To explore the applicability of RosCOS markers in other rosaceous crops, a subset of the RosCOS primers was employed to amplify Malus, Prunus and Fragaria DNA. Malus and Prunus are phylogenetically closer than Fragaria as the former two belong to the same subfamily (Table 1). Despite the larger distance and the multiple SNP between the species, using EST information from all three genera enabled the development of primers that resulted in successful amplification of more than half of the RosCOS in each genus (Table 5). Amplification failures were likely due to the difficulty in designing primers with less than two mismatches in all three genera and presence of a large intron. The amplification success rate remained approximately the same when only two genera contributed to the RosCOS. However, when a RosCOS was represented by two genera, the lowest amplification was observed in the genus for which no EST contributed to the RosCOS. Yet, even when only Prunus EST information is used, the amplification success rate was $77 \%$ in Malus and 23\% in Fragaria. In general, it is evident from these data that successful amplification across all genera is enhanced when EST from two genera contributed to the RosCOS and primer design.

Table 5: Amplification success of RosCOS primers in different genera.

\begin{tabular}{|c|c|c|c|c|}
\hline $\begin{array}{l}\text { Genera represented in } \\
\text { each RosCOS }\end{array}$ & RosCOS per Group & Amplification in Malus & Amplification in Fragaria & $\begin{array}{c}\text { Amplification in Prunus } \\
\text { (cherry) }\end{array}$ \\
\hline Fragaria, Malus and Prunus & 7 & 7 (100\%) & $4(57 \%)$ & $4(57 \%)$ \\
\hline Fragaria and Prunus & 13 & $8(61 \%)$ & $1 \mathrm{I}(85 \%)$ & $10(77 \%)$ \\
\hline Fragaria and Malus & 10 & 7 (70\%) & $10(100 \%)$ & $6(60 \%)$ \\
\hline Prunus and Malus & 18 & $16(89 \%)$ & $9(50 \%)$ & $15(83 \%)$ \\
\hline Prunus & 13 & $10(77 \%)$ & $3(23 \%)$ & $13(100 \%)$ \\
\hline
\end{tabular}




\section{Conclusion}

Comparative genome analysis for the Rosaceae family lags behind that of other economically important families such as the Solanaceae and Poaceae. The RosCOS resource developed in this study aims to ameliorate this situation by providing a marker set that can be employed for comparative mapping and marker development as well as whole genome comparative analyses in the Rosaceae family. The extensive colinearity observed between poplar and Prunus demonstrates the possibility of additional marker development in targeted regions of the Prunus genome based on synteny with poplar. Moreover, with the advent of Rosaceae species whole genome sequence information that will become available in the near future, these RosCOS will be instrumental to place unlinked scaffolds onto genetic maps and enable marker development to targeted regions in species whose genome is not sequenced. Excellent genetic maps and whole genome sequence data are extremely important for QTL discovery and validation. Therefore, the RosCOS resource developed herein has great potential to benefit rosaceaous crop improvement.

\section{Methods \\ Identification of the Rosaceae COS (RosCOS) set}

The set of 3,790 Arabidopsis single copy genes was selected as previously described [33]. The complete data set of 412,827 Rosaceae ESTs as of December 2007 was downloaded from NCBI GenBank [34] and compared to the Arabidopsis single copy gene set using the BLASTX function at the cutoff E-value of 1e-15. The resulting Rosaceae ESTs were assembled using the Contig Assembly Program: CAP3 [35] with parameters of at least 80 bp overlap and $90 \%$ sequence identity. This resulted in the assembly of 7,247 unigenes $(3,818$ contigs and 3,429 singletons) (Figure 1). The 3,818 contigs were assigned a RosCOS number whereas the singletons were not. The consensus sequence for each RosCOS is found under the name ROSC_FMLY_CSA1_1 beginning with RosCOS 1 [36]. The ESTs that are part of the RosCOS are found in the "December 2007 Assembly Info" [37]. The list of single copy Arabidopsis genes and corresponding RosCOS are found under the "December 2007 BLAST info" links [37]. Information about the final list of RosCOS used in this study can be found under the "RosCOS final selection and QC BLAST" links [37]. RosCOS map and primer data is also available from our own database [38] as well as in Additional file 2. Sequence data of the peach parent 'Earlygold' has been deposited in GSS at Genbank [34] and the corresponding accession numbers are listed in Additional file 2 (sheet 2 ).

\section{Design of PCR primers flanking introns}

Orthologous genes share conserved structures such that the position of the introns is conserved [39]. To reduce the probability of sequencing errors in the ESTs and to increase the amplification success rate in multiple Rosaceae species, singletons were discarded from the analysis. Rosaceae contigs comprised of ESTs from three (Fragaria, Malus and Prunus) and two (Fragaria-Malus, PrunusFragaria, and Malus-Prunus) genera as well as only Prunus ESTs were selected totaling up to 1,039 RosCOS that were further investigated. The RosCOS were aligned to the Arabidopsis genome and putative intron sites were identified using the Python Contig Viewer program [40] (Figure 3). Based on the RosCOS sequence length and predicted intron position of these 1039 RosCOS, 857 intron-flanking primer pairs were developed using Primer3 v0.4.0 [41]. Subsequently, all forward primers were designed with an additional M13 tail (CACGACGTTGTAAAACGAC) at the $5^{\prime}$ end to facilitate high-throughput direct sequencing of the amplicons.

\section{PCR conditions and polymorphism detection of RosCOS}

The RosCOS putative intron-flanking primers were used to amplify the peach parent 'Earlygold', $\mathrm{F}_{1}$ and 6 bin set individuals selected from the Prunus TxE $\mathrm{F}_{2}$ reference population $[10,30]$. The amplification reactions were conducted in 96-well plate format in $60 \mathrm{ul}$ reaction volume consisting of $10 \mathrm{mM}$ Tris- $\mathrm{Cl} \mathrm{pH} \mathrm{8.3,50} \mathrm{mM} \mathrm{KCl,} 2 \mathrm{mM}$ $\mathrm{MgCl}_{2}, 10-100 \mathrm{ng}$ of genomic DNA, $0.1 \mathrm{mM}$ of each dNTP, $0.1 \mathrm{uM}$ of each primer, and $0.25 \mathrm{U}$ Taq polymerase. The reactions were preheated at $94^{\circ} \mathrm{C}$ for $1 \mathrm{~min}$ followed by 31 cycles of $92^{\circ} \mathrm{C}(30 \mathrm{~s}), 56^{\circ} \mathrm{C}(30 \mathrm{~s}), 72^{\circ} \mathrm{C}(30 \mathrm{~s})$, and a final extension of $72^{\circ} \mathrm{C}(60 \mathrm{~s})$. Amplified fragments were sequenced using the M13F primer at the Agencourt Bioscience Corporation (Agencourt, Beverly, MA, USA). The sequencing results were analyzed for polymorphisms such as single nucleotide polymorphism (SNP) and/or insertion-deletions (InDels) using Sequencher software v4.2 (Gene Codes Corporation). The presence of a double peak in an otherwise high-quality chromatogram was indicative of the presence of a SNP. The sudden decay of high-quality chromatogram was indicative of the presence of an InDel.

\section{Genotyping and Mapping}

Bins representing the different regions of the Prunus genome have been identified by the genotype of a subset of plants from the TxE $\mathrm{F}_{2}$ population [30]. RosCOS markers with a segregation pattern corresponding to a bin set score were grouped in that bin. RosCOS that mapped in bin $2: 45$ or $3: 04$ and $5: 41$ or $8: 30$, respectively, were analyzed in a $7^{\text {th }}$ genotype to map them in one or the other bin. RosCOS markers that clearly segregated but did not fall into a known bin were categorized as "orphan" RosCOS markers.

\section{Synteny of RosCOS and Poplar COS}

The translated sequence of Arabidopsis single copy genes corresponding to the bin-mapped RosCOS were compared to the Populus trichocarpa genome. Using the P. tri- 
chocarpa $v 1.1$ genome browser [42], the physical position of each poplar COS was identified through the TBLASTN function with the cut off E-value of 1e-5. Syntenic blocks between Prunus and poplar were established under the condition that a minimum of three linked RosCOS corresponded to poplar COS that were located within $2 \mathrm{Mb}$ from each other.

\section{Authors' contributions}

AC performed the intron-flanking primer design, the amplification reactions, the bin mapping experiments, the comparative analysis with poplar and Arabidopsis, and analyzed the data. AK developed the pipeline for the RosCOS identification. WH and PA provided the DNAs from the TxE bin set and critically evaluated the analysis of the bin mapping data. AI provided overall advice and coordination of this project, and participated in the supervision. EV conceived the RosCOS idea, designed and supervised the study. AC and $\mathrm{EV}$ wrote the manuscript with edits from the other coauthors. All authors read and approved the final manuscript.

\section{Additional material}

\section{Additional file 1}

Genus representation and primer development of RosCOS.

Click here for file

[http://www.biomedcentral.com/content/supplementary/1471-

2164-10-562-S1.doc]

\section{Additional file 2}

RosCOS marker, map, primer, SNP and InDel, accession number information.

Click here for file

[http://www.biomedcentral.com/content/supplementary/1471-

2164-10-562-S2.xls]

\section{Additional file 3}

Map location of two or more RosCOS identified by one Arabidopsis single copy gene. Dataset shows the map position of the putatively duplicated COS.

Click here for file

[http://www.biomedcentral.com/content/supplementary/14712164-10-562-S3.doc]

\section{Acknowledgements}

This work is supported by USDA-NRI grants 2008-02259 and 2005-00743. AC was also supported by funds from the Department of Horticulture and Crop Science, The Ohio State University. The authors would like to thank Drs. Dan Sargent and David Chagne for providing DNAs from strawberry and apple, respectively.

\section{References}

I. Potter D, Eriksson T, Evans RC, Oh S, Smedmark JEE, Morgan DR, Kerr M, Robertson KR, Arsenault M, Dickinson TA, Campbell CS: Phylogeny and classification of Rosaceae [electronic resource]. Plant Syst Evol 2007, 266:5-43.
2. Shulaev V, Korban SS, Sosinski B, Abbott AG, Aldwinckle HS, Folta KM, lezzoni A, Main D, Arus P, Dandekar AM, Lewers K, Brown SK, Davis TM, Gardiner SE, Potter D, Veilleux RE: Multiple models for Rosaceae genomics. Plant Physiol 2008, 147:985-1003.

3. Tanksley SD, Bernatzky R, Lapitan NL, Prince JP: Conservation of gene repertoire but not gene order in pepper and tomato. Proc Natl Acad Sci USA 1988, 85:6419-6423.

4. Livingstone KD, Lackney VK, Blauth JR, van Wijk R, Jahn MK: Genome Mapping in Capsicum and the Evolution of Genome Structure in the Solanaceae. Genetics 1999, I 52:1 I83-1 202.

5. Bonierbale MW, Plaisted RL, Tanksley SD: RFLP Maps Based on a Common Set of Clones Reveal Modes of Chromosomal Evolution in Potato and Tomato. Genetics 1988, 1 20:1095-I 103.

6. Devos KM, Gale MD: Comparative genetics in the grasses. Plant Mol Biol 1997, 35:3-I5.

7. Paterson AH, Bowers JE, Burow MD, Draye X, Elsik CG, Jiang CX, Katsar CS, Lan TH, Lin YR, Ming R, Wright RJ: Comparative genomics of plant chromosomes. Plant Cell 2000, I 2: I 523-I 540.

8. Yan L, Loukoianov A, Tranquilli G, Helguera M, Fahima T, Dubcovsky J: Positional cloning of the wheat vernalization gene VRNI . Proc Natl Acad Sci USA 2003, 100:6263-6268.

9. Dirlewanger E, Graziano E, Joobeur T, Garriga-Caldere F, Cosson P, Howad W, Arus P: Comparative mapping and marker-assisted selection in Rosaceae fruit crops. Proc Natl Acad Sci 2004, 101:9891-9896.

10. Joobeur T, Viruel MA, de Vicente MC, Jauregui B, Ballester J, Dettori MT, Verde I, Truco MJ, Messeguer R, Batlle I, Quarta R, Dirlewanger $E$, Arus $P$ : Construction of a saturated linkage map for Prunus using an almond $x$ peach F2 progeny. Theor Appl Genet 1998, 97:1034-104I.

II. Lambert P, Hagen LS, Arus P, Audergon JM: Genetic linkage maps of two apricot cultivars (Prunus armeniaca $L$.) compared with the almond Texas $\times$ peach Earlygold reference map for Prunus. Theor Appl Genet 2004, I 08: I I 20- I 130.

12. Olmstead JW, Sebolt AM, Cabrera A, Sooriyapathirana SS, Hammar S, Iriarte G, Wang D, Chen CY, Knaap E van der, lezzoni AF: Construction of an intra-specific sweet cherry (Prunus avium L.) genetic linkage map and synteny analysis with the Prunus reference map. Tree Genet Genomes 2008, 4:897-910.

13. Clarke JB, Sargent DJ, Boškoviæ RI, Belaj A, Tobutt KR: A cherry map from the inter-specific cross Prunus avium 'Napoleon' $x$ $P$. nipponica based on microsatellite, gene-specific and isoenzyme markers. Tree Genet Genomes 2009, 5:4I-5I.

14. Dirlewanger E, Cosson P, Howad W, Capdeville G, Bosselut N, Claverie M, Voisin R, Poizat C, Lafargue B, Baron O, Laigret F, Kleinhentz $M$, Arús $P$, Esmenjaud $D$ : Microsatellite genetic linkage maps of myrobalan plum and an almond-peach hybrid--location of root-knot nematode resistance genes. Theor Appl Genet 2004, 109:827-838.

15. Dondini L, Lain O, Geuna F, Banfi R, Gaiotti F, Tartarini S, Bassi D, Testolin R: Development of a new SSR-based linkage map in apricot and analysis of synteny with existing Prunus maps. Tree Genet Genomes 2007, 3:239-249.

16. Sargent DJ, Rys A, Nier S, Simpson DW, Tobutt KR: The development and mapping of functional markers in Fragaria and their transferability and potential for mapping in other genera. Theor Appl Genet 2007, I I 4:373-384.

17. Gasic K, Han Y, Kertbundit S, Shulaev V, lezzoni A, Stover E, Bell R, Wisniewski M, Korban S: Characteristics and transferability of new apple EST-derived SSRs to other Rosaceae species. Mol Breeding 2009, 23:397-4II.

18. Sargent DJ, Marchese A, Simpson DW, Howad W, Fernandez-Fernandez F, Monfort A, Arus P, Evans KM, Tobutt KR: Development of "universal" gene-specific markers from Malus spp. cDNA sequences, their mapping and use in synteny studies within Rosaceae. Tree Genet Genomes 2009, 5: I33-145.

19. Vilanova S, Sargent DJ, Arus P, Monfort A: Synteny conservation between two distantly-related Rosaceae genomes: Prunus (the stone fruits) and Fragaria (the strawberry). BMC Plant Biol 2008, 8:67.

20. Fulton TM, Hoeven R Van der, Eannetta NT, Tanksley SD: Identification, analysis, and utilization of conserved ortholog set markers for comparative genomics in higher plants. Plant Cell 2002, 14:1457-1467.

21. Wu F, Mueller LA, Crouzillat D, Petiard V, Tanksley SD: Combining bioinformatics and phylogenetics to identify large sets of sin- 
gle-copy orthologous genes (COSII) for comparative, evolutionary and systematic studies: a test case in the euasterid plant clade. Genetics 2006, I74: 1407-1420.

22. McCouch SR: Genomics and Synteny. Plant Physiol 200I, 125:152-155.

23. Liewlaksaneeyanawin C, Zhuang J, Tang M, Farzaneh N, Lueng G, Cullis C, Findlay S, Ritland CE, Bohlmann J, Ritland K: Identification of COS markers in the Pinaceae. Tree Genet Genomes 2009, 5:247-255.

24. Chapman MA, Chang J, Weisman D, Kesseli RV, Burke JM: Universal markers for comparative mapping and phylogenetic analysis in the Asteraceae (Compositae). Theor Appl Genet 2007, I I 5:747-755.

25. Krutovsky KV, Elsik CG, Matvienko M, Kozik A, Neale DB: Conserved ortholog sets in forest trees. Tree Genet Genomes 2006, 3:6I-70.

26. Wu F, Eannetta N, Xu Y, Tanksley S: A detailed synteny map of the eggplant genome based on conserved ortholog set II (COSII) markers. TAG Theoretical and Applied Genetics 2009, I I 8:927-935.

27. Wu F, Eannetta N, Xu Y, Durrett R, Mazourek M, Jahn M, Tanksley S: A COSII genetic map of the pepper genome provides a detailed picture of synteny with tomato and new insights into recent chromosome evolution in the genus Capsicum. TAG Theoretical and Applied Genetics 2009, I I 8:1279-1293.

28. Timms L, Jimenez R, Chase M, Lavelle D, McHale L, Kozik A, Lai Z, Heesacker A, Knapp S, Rieseberg L, Michelmore R, Kesseli R: Analyses of synteny between Arabidopsis thaliana and species in the Asteraceae reveal a complex network of small syntenic segments and major chromosomal rearrangements. Genetics 2006, 173:2227-2235.

29. Krutovsky KV, Troggio M, Brown GR, Jermstad KD, Neale DB: Comparative mapping in the Pinaceae. Genetics 2004, 168:447-46I.

30. Howad W, Yamamoto T, Dirlewanger E, Testolin R, Cosson P, Cipriani G, Monforte AJ, Georgi L, Abbott AG, Arus P: Mapping with a few plants: using selective mapping for microsatellite saturation of the Prunus reference map. Genetics 2005, | 7 I: | 305-1309.

31. Tuskan GA, Difazio S, Jansson S, Bohlmann J, Grigoriev I, Hellsten U, Putnam N, Ralph S, Rombauts S, Salamov A, Schein J, Sterck L, Aerts A, Bhalerao RR, Bhalerao RP, Blaudez D, Boerjan W, Brun A, Brunner A, Busov V, Campbell M, Carlson J, Chalot M, Chapman J, Chen GL, Cooper D, Coutinho PM, Couturier J, Covert S, Cronk Q, Cunningham R, Davis J, Degroeve S, Dejardin A, Depamphilis C, Detter J, Dirks B, Dubchak I, Duplessis S, Ehlting J, Ellis B, Gendler K, Goodstein D, Gribskov M, Grimwood J, Groover A, Gunter L, Hamberger B, Heinze B, Helariutta Y, Henrissat B, Holligan D, Holt R, Huang W, Islam-Faridi N, Jones S, Jones-Rhoades M, Jorgensen R, Joshi C, Kangasjarvi J, Karlsson J, Kelleher C, Kirkpatrick R, Kirst M, Kohler A, Kalluri U, Larimer F, Leebens-Mack J, Leple JC, Locascio P, Lou Y, Lucas S, Martin F, Montanini B, Napoli C, Nelson DR, Nelson C, Nieminen K, Nilsson O, Pereda V, Peter G, Philippe R, Pilate G, Poliakov A, Razumovskaya J, Richardson P, Rinaldi C, Ritland K, Rouze P, Ryaboy D, Schmutz J, Schrader J, Segerman B, Shin H, Siddiqui A, Sterky F, Terry A, Tsai C], Uberbacher E, Unneberg P, Vahala J, Wall K, Wessler S, Yang G, Yin T, Douglas C, Marra M, Sandberg G, Peer Y Van de, Rokhsar D: The genome of black cottonwood, Populus trichocarpa (Torr. \& Gray). Science 2006, 3 I 3:1596-1604.

32. Jung S, Jiwan D, Cho I, Lee T, Abbott A, Sosinski B, Main D: Synteny of Prunus and other model plant species. BMC Genomics 2009, 10:76.

33. Van Deynze A, Stoffel K, Buell CR, Kozik A, Liu J, Knaap E van der, Francis D: Diversity in conserved genes in tomato. BMC Genomics 2007, 8:465.

34. National Center for Biotechnology Information [http:// www.ncbi.nlm.nih.gov/]

35. Huang $X$, Madan A: CAP3: A DNA sequence assembly program. Genome Res 1999, 9:868-877.

36. RosCOS consensus sequence [http://cgpdb.ucdavis.edu/ rosaceae assembly/ rosaceae sequences 412832 Dec 2007.Clean.COS.CDS.assembly]

37. RosCOS Assembly [http://cgpdb.ucdavis.edu/rosaceae assembly/]

38. RosCOS map and primer database [http://bioinfo.bch.msu.edul rosaceae cos]
39. Fedorov A, Merican AF, Gilbert W: Large-scale comparison of intron positions among animal, plant, and fungal genes. Proc Natl Acad Sci USA 2002, 99:16128-16133.

40. Contig Viewer Program [http://www.atgc.org/Py ContigViewer]

41. Primer3 (v. 0.4.0) [http://frodo.wi.mit.edu/primer3/]

42. Populus trichocarpa vl.I [http://genome.jgi-psf.org/Poptrl I/ Poptrl I.home.html]
Publish with Biomed Central and every scientist can read your work free of charge

"BioMed Central will be the most significant development for disseminating the results of biomedical research in our lifetime. "

Sir Paul Nurse, Cancer Research UK

Your research papers will be:

- available free of charge to the entire biomedical community

- peer reviewed and published immediately upon acceptance

- cited in PubMed and archived on PubMed Central

- yours - you keep the copyright 